\title{
Capturing the Relative Distribution of Features for Action Recognition
}

\author{
Olusegun Oshin Andrew Gilbert Richard Bowden \\ Centre for Vision, Speech and Signal Processing, \\ University of Surrey \\ Guildford, Surrey \\ United Kingdom GU2 7XH \\ \{o.oshin, a.gilbert, r.bowden\}esurrey.ac.uk
}

\begin{abstract}
This paper presents an approach to the categorisation of spatio-temporal activity in video, which is based solely on the relative distribution of feature points. Introducing a Relative Motion Descriptor for actions in video, we show that the spatio-temporal distribution of features alone (without explicit appearance information) effectively describes actions, and demonstrate performance consistent with stateof-the-art. Furthermore, we propose that for actions where noisy examples exist, it is not optimal to group all action examples as a single class. Therefore, rather than engineering features that attempt to generalise over noisy examples, our method follows a different approach: We make use of Random Sampling Consensus (RANSAC) to automatically discover and reject outlier examples within classes. We evaluate the Relative Motion Descriptor and outlier rejection approaches on four action datasets, and show that outlier rejection using RANSAC provides a consistent and notable increase in performance, and demonstrate superior performance to more complex multiplefeature based approaches.
\end{abstract}

\section{INTRODUCTION}

Human action recognition from video has gained significant attention in the field of Computer Vision. The ability to automatically recognise actions is important because of potential applications in video indexing and search, activity monitoring for surveillance, and assisted living purposes. The task is especially challenging due to variations in factors pertaining to video setup and execution of the actions. These include illumination, scale, camera motion, viewpoint, background, occlusion, action length, subject appearance and style. While excellent results have been obtained on simulated actions in simplified settings, natural actions in uncontrolled environments, such as movies and personal video collections, as can be seen in figure 1, have proven more difficult.

Recent approaches to action recognition in natural video argue that both shape and motion information are necessary for recognition. They often combine static image features and spatio-temporal features to capture appearance and motion. It has, however, been shown in psychology experiments on Point Light Displays [1] that it is possible to recognise human actions based entirely on the dynamics of body movement. This gives motivation for our approach. Our approach is further motivated by the fact that, the appearance of subjects or background is less important to the description of actions than dynamics. In this paper, we investigate the sole use of body dynamics for automatic action recognition

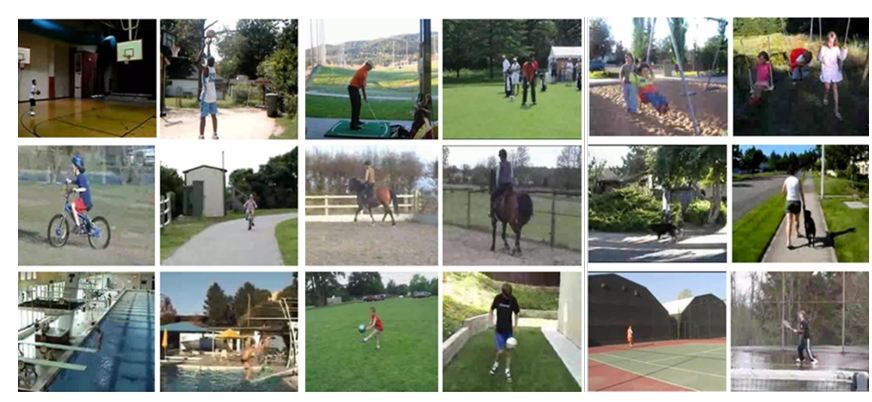

Fig. 1. Selected example actions of the YouTube human action dataset, showing the complex nature of actions in uncontrolled environments.

in complex videos. We propose a novel representation of actions in video, which captures the relative distribution of motion-based interest points by encoding their local spatiotemporal configuration in an efficient manner. This results in an action descriptor, which, in vectorised form, can be learnt using SVMs.

Furthermore, while current methods treat all examples of a particular action as one class and seek generalisation across all examples, we propose the use of Random Sampling Consensus (RANSAC) [2] to automatically split training class examples into inlier and outlier subdivisions in order to identify examples with significant within-class variations. These noisy examples may prove detrimental to the overall classifier performance, and we propose that by identifying and rejecting them, classification performance can be improved.

The layout for the remainder of this paper is as follows: Section II discusses related research. In Section III, we present our approach in detail, giving an overview of the Relative Motion Descriptor (RMD). We describe our experimental setup in Section V and present recognition results in Section VI. Finally, Section VII concludes the paper.

\section{RELATED WORK}

There is a considerable body of work exploring the description of actions in video for recognition. Many of the approaches make use of a sparse set of local interest points generated by the action [3], [4], [5], [6], [7], [8], and have demonstrated remarkable performance. Interest points are highlighted as salient regions based on response functions 
applied to the video, and eliminate the need for motion tracking and background subtraction, which are costly preprocessing steps. Examples include Laptev and Lindeberg's [9] temporal extension of Harris 2D corners, Willems et al's [10] use of the determinant of the generalised 3D Hessian matrix, and Dollar et al.'s [5] separable linear filters.

Several descriptors have been proposed to encode the spatio-temporal support region of these interest points. These include local jet descriptors [3], vector of concatenated pixel gradients [5], generalisation of the SIFT and SURF descriptors [11], [12], [10], and Laptev et al.'s [6] HOG/HOF descriptor. Detected interest points are typically used in a discriminative [3], [5] or generative [4] model.

While earlier action recognition methods were evaluated on simulated actions in simplified settings, more recent work has shifted focus to natural actions in unconstrained scenarios, e.g. personal video collections and movies. As a result of the increase in complexity of these actions, recent approaches [13], [6], [14] make use of a combination of feature types. In contrast, our approach utilises one feature type.

A number of recent approaches have shown good recognition performance by capturing the local spatio-temporal configuration of interest points. Gilbert et al. [8] build compound hierarchical features based on relationships of detected interest points, and use data mining to discover reoccurring patterns. Ryoo and Aggarwal [15] use a set of pairwise predicates to describe relationships between interest points, while Matikainen et al. [16] build relative location probability maps of interest points. Kovashka et al. [17] construct a hierarchy of vocabularies from spatio-temporal neighbourhoods of interest points, encoding the points and a configuration of their neighbours. Savarese et al. [18] also capture pairwise correlation of interest point labels based on their proximity. While these methods encode configurations based on the appearance and location of interest points, our approach makes use of their locations and strengths only, discarding appearance information.

\section{ACTION ENCODING}

The aim of this work is to discriminate between actions in complex scenes using the dynamics of body movements as captured by spatio-temporal interest points. In contrast to other methods tackling action recognition in complex scenes, we present a generic method that utilises the distribution of motion-induced features only. Actions can be described in terms of the motion of subjects and the objects with which they interact. A large portion of the motion may be localised with respect to the subject, as is observed with a Clapping or Answer Phone action, while the motion of other actions are holistic, and may include background motion, e.g. Running, Horseback Riding, etc. For the Clapping and Answer Phone actions, the response of interest point detectors will generally be greater along the arms than at other parts. Also, the spatial and temporal distribution of the interest points induced by the motion of the arms provide discriminative information for action description.

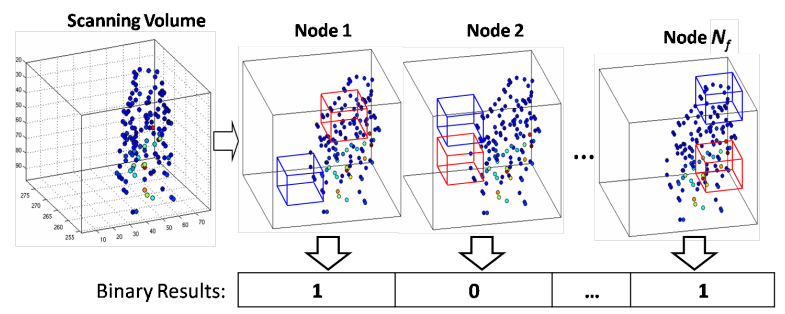

(a) A spatio-temporal snapshot of an action in terms of its interest points, also showing the regions that are compared within a scanning volume.

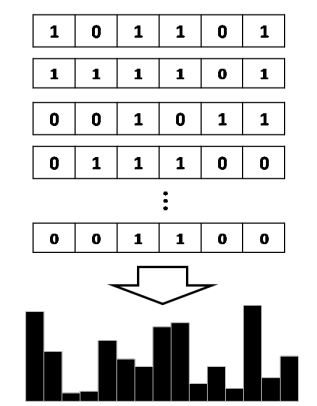

(b) A sequence of binary results obtained from the node tests in (a) above, which are modelled with a histogram

Fig. 2. The Relative Motion Descriptor.

In order to capture this information, we encode the local spatio-temporal configuration of interest points. We make use of binary tests which measure the relative strengths of interest point responses as given by an interest point detector. In contrast to Ryoo and Aggarwal [15], who construct histograms of particular pairwise relationships using a set of spatial (near, xnear, ynear, far) and temporal (equals, meets, before, overlaps, etc) predicates, our method encodes the relative strengths of interest point responses (if any) between two regions, in a scanning window approach. This section describes our method.

\section{A. The Relative Motion Descriptor (RMD)}

We propose an action descriptor inspired by the Randomised Ferns ensemble classifier [19], which are nonhierarchical classification structures derived from Random Forests. Each randomised fern consists of set of ordered binary tests, which split the space of data being classified.

Our approach makes use of these ordered tests. However, in contrast to Randomised Ferns and Forests which are classifiers, we use the representation to create an action descriptor, which is then used in conjunction with a more powerful non-linear Support Vector Machine classifier. This captures the local spatio-temporal dependencies of the motion in a scanning volume approach.

We define a set of tests $f_{j}, j=\left\{1 \ldots N_{f}\right\}$, where $N_{f}$ is the number of tests. For a particular instance of a scanning volume $i_{X Y T}$ with dimensions $X, Y, T$ taken from a video $I$, we define our test as a comparison between two regions randomly positioned within the scanning volume at points $x, y, t$. Given that the regions have spatial and temporal extents $\frac{X}{\sigma}, \frac{Y}{\sigma}, \frac{T}{\tau}$, and $s_{i}$ is the sum of interest point response 
strengths within a region $i$, the result of a binary node test $f_{j}$ is given by,

$$
f_{j}= \begin{cases}1 & s_{1}<s_{2} \\ 0 & \text { otherwise }\end{cases}
$$

The sum of interest point response strengths, $s_{i}$ is given by

$$
s\left(x, y, t, \frac{X}{\sigma}, \frac{Y}{\sigma}, \frac{T}{\tau}\right)=\sum_{x^{\prime}=x}^{x+\frac{X}{\sigma}} \sum_{y^{\prime}=y}^{y+\frac{Y}{\sigma}} \sum_{t^{\prime}=t}^{t+\frac{T}{\tau}} \imath\left(x^{\prime}, y^{\prime}, t^{\prime}\right) .
$$

where $\imath$ is the representation of the video in terms of detected interest points only, given by

$$
\imath(x, y, t)= \begin{cases}\Re\left(I_{x y t}\right) & \text { if } \Re\left(I_{x y t}\right)>\Gamma \\ 0 & \text { otherwise. }\end{cases}
$$

$\Re$ is the strength of the response function of an interest point detector, applied to the video at point $x, y, t$, and $\Gamma$ is the threshold above which interest points are detected. $\Gamma$ can be varied so that either dense or sparse interest points are detected.

Results of node tests can be encoded using higher radixes, e.g, ternary or quaternary, instead of binary, hence quantizing the node test results with more symbols. This allows additional information about the distribution of interest points to be captured, without increasing the number of tests. To this end, equation 1 above can be modified such that the node test result, $f_{j}$, is given by,

$$
f_{j}= \begin{cases}2 & \text { if }\left(s_{1}-s_{2}\right) \geq \hbar \times\left(s_{1}-s_{2}\right) \\ 1 & \text { if }\left(s_{2}-s_{1}\right) \geq \hbar \times\left(s_{2}-s_{1}\right) \\ 0 & \text { otherwise }\end{cases}
$$

for ternary tests, or

$$
f_{j}= \begin{cases}3 & \text { if }\left(s_{1}-s_{2}\right)>\hbar \times\left(s_{1}-s_{2}\right) ; \\ 2 & \text { if } 0<\left(s_{1}-s_{2}\right) \leq \hbar \times\left(s_{1}-s_{2}\right) \\ 1 & \text { if } 0 \leq\left(s_{2}-s_{1}\right) \leq \hbar \times\left(s_{2}-s_{1}\right) \\ 0 & \text { otherwise }\end{cases}
$$

for quaternary tests, where $0<\hbar<1$ can be arbitrarily chosen.

At each position of the scanning volume, we obtain $\left\{f_{1}, f_{2}, \ldots, f_{N_{f}}\right\}$, which can be concatenated to form a binary, ternary or quaternary value of length $N_{f}$, which is in turn converted into a decimal code, $\delta$ given by,

$$
\delta_{i_{X Y T}}=\sum_{j}^{N_{f}} f_{j} \times n^{j}
$$

where $n$ is the number of symbols.

The scanning window response, $\delta$ is modelled with a histogram of size $n^{N_{f}}$. Additional tests can be performed by repeating this process for the same video, and the resulting histograms are concatenated. Figure 2 shows a scanning volume with 6 nodes and depicts how the results of the node comparisons are combined in a binary fashion to form the histogram.

\section{Automatic Outlier Detection via Random SAMPLING CONSENSUS}

Having assembled concatenated histograms of relative distribution that capture the local spatio-temporal configuration of interest points over a video, SVMs can be used to learn discriminative classifiers from the representation. Since there exists noise and variation in the execution of natural actions and the capture conditions in movies and personal videos, examples will be present in training which are detrimental to the performance of the classifiers. While other classification approaches attempt to learn generalisation over these noisy examples, we argue that grouping all examples into one semantic class limits the performance of the classifier. We propose the application of RANSAC [2] to automatically detect and discard noisy training examples.

Given a set of training examples $\Phi$ belonging to a particular class $C$ on which RANSAC outlier rejection is to be performed, we iteratively select a random subset, $\varphi \subset \Phi$ of the examples. We then train a binary SVM classifier of the subset $\varphi$ against all training examples from other classes. Testing is then performed on the remainder of the training example set, $\psi \subset \Phi$, where $\Phi=\varphi \cup \psi$. After several iterations of this random training and testing, we select the training subset $\varphi$ that results in the highest number of correctly classified examples in subset $\psi$, labelled $\varsigma$. For the class in question, $C$, inliers are chosen as $\varphi \cup \varsigma$, while outliers are $\psi-\varsigma$. After RANSAC is applied, the outlier subset of the training examples is discarded, and the inliers form the training set for class $C$.

Since the subset $\varphi$ is trained against training examples from other classes, the procedure identifies examples in class $C$ that are most likely to cause confusion with other classes. Therefore, the discarding of these examples simplifies the modelling of actions by the classifier. In SVMs, for example, the soft margin extension permits misclassifications via the use of slack variables for non-separable training sets. Since our method discards detected outliers, the slack variable and hence the training error are reduced. We hypothesise that this allows for improved class separability. The novel application of RANSAC in this domain is the second contribution of this paper.

\section{EXPERIMENTAL SETUP}

We apply the RMD to actions in video by running a scanning volume over the video. To do this efficiently, we sub-sample all example videos to $120 \times 160$ pixels spatially. Since we do not make use of explicit appearance information and detected interest points are based on motion, the information lost as a result of sub-sampling is negligible. We also compute integral volume representations of detected interest points to efficiently compute the sums $s_{i}$ for the descriptor (section III-A).

For our RANSAC implementation, the size of the training subset, $\varsigma$ is chosen as one-third of the number of training examples, $\Phi$. We investigated the effect of changing the size of the scanning volume, $i_{X Y T}$, and the size of regions within the scanning volume, given by $\frac{X}{\sigma}, \frac{Y}{\sigma}, \frac{T}{\tau}$. This is done on the 


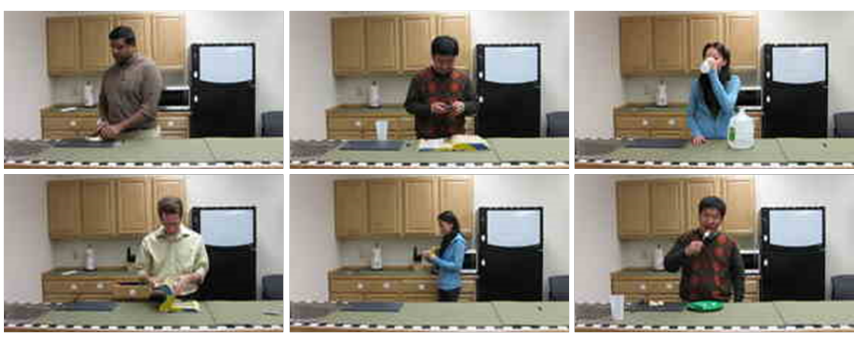

Fig. 3. Selected examples of six of the ADL dataset actions.

training data by evaluating the overall recall of the RANSAC validation examples, $\frac{\varsigma}{\psi}$ across all classes. We perform these parameter selection experiments for all datasets.

For all datasets, we compare $N_{f}=4$ node tests, and use the quaternary representation of the tests (equation 5) with $\hbar=0.01$, resulting in 256-bin histograms. For each video, we concatenate 15 histograms of relative motion, giving a 3840-length vector.

We use a non-linear Support Vector Machine with a Radial Basis Function (RBF) kernel, defined by, $k\left(\mathbf{x}_{i}, \mathbf{x}_{j}\right)=$ $\exp \left(-\gamma\left\|\mathbf{x}_{i}-\mathbf{x}_{j}\right\|^{2}\right)$, where $\mathbf{x}_{i}$ and $\mathbf{x}_{j}$ represent concatenated histograms of relative motion. We construct a multi-class classifier by combining binary classifiers in a one-againstrest training scheme. Each test example is classified by all binary classifiers, returning a measure of confidence. The classifier that returns the highest confidence is chosen.

\section{A. Interest Point Detection}

We generate interest points from action videos with the widely used interest point detector of Dollar et al. [5]. Dollar applies a response function to the video of the form, $\mathfrak{R}=(I *$ $\left.g * h_{e v}\right)^{2}+\left(I * g * h_{o d}\right)^{2}$, where $g(x, y: \sigma)$ is the 2D Gaussian kernel applied along the spatial dimensions of the video, and $h_{e v}$ and $h_{o d}$ are a pair of 1D Gabor filters applied in the temporal dimension. Local maxima of the response function $\Re$ above the threshold $\Gamma$ (See equation 3 ), are selected as interest points. It should be noted that our method is generic and is not dependent the interest point detection method.

\section{B. Datasets}

The KTH dataset [3] contains videos of 25 persons performing six actions in four different conditions. These actions are Boxing, Clapping, Waving, Jogging, Running and Walking. The conditions include scale variations, different clothes and lighting changes. The Assisted Daily Living (ADL) dataset [20] consists of high resolution videos of activities performed in daily living. Actions include Answer Phone, Chop Banana, Dial Phone, Look Up In Directory, Write On Whiteboard, Drink Water, Eat Snack, Peel Banana, Eat Banana, and Eat With Silverware. These actions were chosen for their similarity and the difficulty in separating between actions using only one feature type. Figure 3 shows examples of the dataset.

While both of the datasets above contain simulated actions performed in the presence of minimum background clutter,

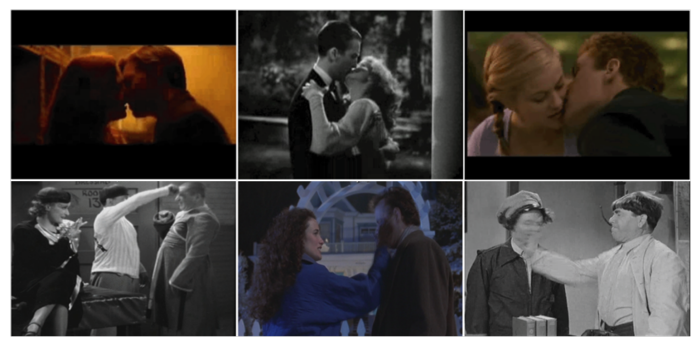

Fig. 4. Selected examples of the Kisses/Slaps dataset actions.

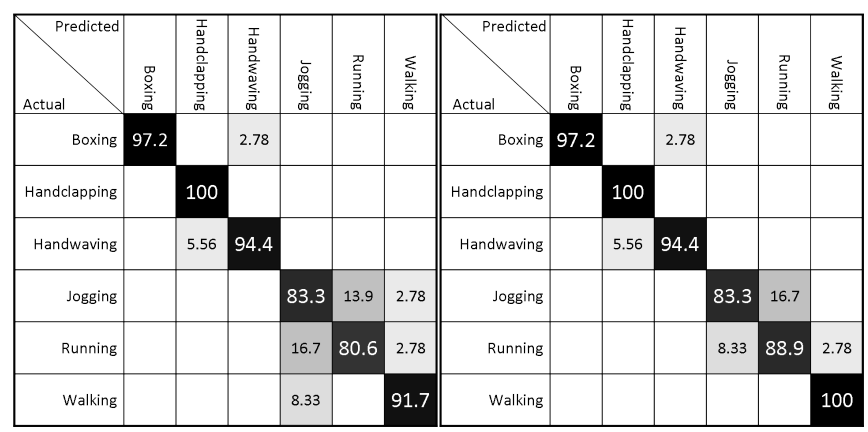

Fig. 5. Confusion Matrices for the RMD on the KTH dataset. Left: without outlier rejection (91.2\%); Right: with RANSAC outlier rejection (94\%).

using static cameras, the Kisses/Slaps dataset [21] contains actions in two classes - Kissing and Hitting - compiled from movies. These actions are performed by different actors, at different scales, and in a wide range of scenes. Examples are show in Figure4. The YouTube dataset [7] contains actions obtained from YouTube, TV broadcast, and personal video collections and are captured under uncontrolled conditions. The videos are of varying resolution, and contain significant variation. There are 11 action categories as listed in Table IV. A subset can be seen in Figure 1.

For the purpose of comparison, we use the Training/Validation/Test split for the KTH dataset as outlined in [3], and for the other datasets, we use Leave One Out Cross validation as prescribed.

\section{RESULTS}

For the KTH dataset, using the training/validation/test split defined by [3], we obtain an overall accuracy of $91.2 \%$ with our RMD, which is consistent with the state-of-theart. The most confusion is observed between Jogging and Running as they appear very similar. With the application of RANSAC, the overall accuracy is improved to $94 \%$, which is equivalent to the best results reported on this dataset [22]. This is remarkable as only one feature type is used, and no explicit appearance information is encoded by the RMD. Table I compares results with other methods. Given that the interest point detection method of Dollar et al. is used, our approach achieves considerably better performance than [5]. This shows that the majority of the discriminative power is less to do with feature types, but the nature of information obtained from them. 


\begin{tabular}{|c|c|}
\hline Method & Accuracy \\
\hline Schuldt et al. [3] & $71.72 \%$ \\
Dollar et al. [5] & $81.2 \%$ \\
Fathi and Mori [23] & $90.5 \%$ \\
Klaser et al. [12] & $91.4 \%$ \\
Marszalek et al. [6] & $91.8 \%$ \\
Kovashka et al. [17] & $94.5 \%$ \\
Gilbert et al. [22] & $94.5 \%$ \\
\hline RMD & $91.2 \%$ \\
RMD + RANSAC & $94.1 \%$ \\
\hline
\end{tabular}

TABLE I

AVERAGE ACCURACIES ON THE KTH DATASET USING THE TRAINING/VALIDATION/TEST SPLIT DEFINED IN [3].

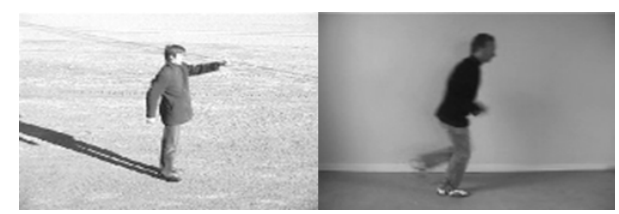

Fig. 6. Examples of outliers selected from the KTH dataset. Left: Boxing action with interest points detected on shadow; Right: Running action that is misclassified during outlier rejection as Jogging.

Figure 5 shows the confusion matrices obtained for the KTH dataset using the RMD with and without outlier rejection, and the effects of removing noisy examples from training can be observed. Having performed the RANSAC procedure on all classes of the dataset, no outliers were obtained for the action Handclapping. Handwaving and Walking had 2 rejected examples each, while 4, 6, and 7 outliers were obtained for Boxing, Running and Jogging respectively. The results show, as in the case of Boxing and Waving, that in some cases the removal of a small number of examples do not affect the class confusion. For Walking, outlier removal results in perfect classification. However, much confusion still exists between the Jogging and Running classes. This is due to the actions being indistinguishable in many of the examples. Figure 6 shows examples of outliers discarded by the RANSAC procedure. It is observed that some outliers are regarded as such because of interest points detected on shadows of the subject, which affect the distribution. For translational actions of the dataset, videos with scale change constitute some of the outliers, while the majority are action examples performed indoors, where the Running action is often performed slower and can be easily confused with Jogging, hence these two classes are shown to produce the most outliers.

To test the generality of the performance of our outlier rejection method on other feature types and descriptors, we apply it to the method of Laptev et al. [6] on the KTH dataset. We obtain HOG/HOF descriptors using the interest point detection method of [9], and train a SVM classifier. Without outlier rejection, we obtain an accuracy of $88.9 \%$ using an RBF kernel (Best results [6] are obtained using a $\chi^{2}$ kernel). With noisy training examples discarded, the accuracy increases to $92.7 \%$. This shows that our approach

\begin{tabular}{|c|c|c|c|c|}
\hline Method & $\begin{array}{c}\text { Action } \\
\mathrm{MACH}[21]\end{array}$ & LTP [24] & RMD & $\begin{array}{c}\text { RMD + } \\
\text { RANSAC }\end{array}$ \\
\hline Kisses & $66.4 \%$ & $77.3 \%$ & $76.1 \%$ & $82.4 \%$ \\
\hline Slaps & $67.2 \%$ & $84.2 \%$ & $73.1 \%$ & $77.2 \%$ \\
\hline Average & $66.8 \%$ & $80.75 \%$ & $74.6 \%$ & $79.8 \%$ \\
\hline
\end{tabular}

TABLE II

COMPARISON ON THE KISSES/SLAPS DATASET.

\begin{tabular}{|c|c|}
\hline Method & Accuracy \\
\hline Velocity Histories [20] & $63 \%$ \\
Latent Velocity Histories [20] & $63 \%$ \\
Tracklets [25] & $82.7 \%$ \\
Augmented Velocity Histories with & \\
Relative and Absolute Position [20] & $89 \%$ \\
\hline RMD & $84 \%$ \\
RMD + RANSAC & $\mathbf{8 9 . 3 \%}$ \\
\hline
\end{tabular}

TABLE III

COMPARISON ON ASSISTED DAILY LIVING DATASET.

benefits existing action recognition methods.

We also present results on the Kisses/Slaps dataset. We obtain an overall accuracy of $74.6 \%$ using our RMD, without outlier rejection. This is a considerable improvement on the Action MACH approach of Rodriguez et al. [21]. With outlier rejection, the accuracy improves to $79.8 \%$, which is $<1 \%$ lower than Local Trinary Patterns [24]. Table II compares results on the Kisses/Slaps dataset.

Without outlier rejection, the overall accuracy obtained for the Assisted Daily Living dataset is $84 \%$. The application of RANSAC results in an improvement in overall accuracy, giving $89.3 \%$. This also shows a slight improvement in performance over Messing et al [20], who obtain an accuracy of $89 \%$. Given the similarity between the motions in the dataset, the result is impressive as no explicit appearance information is used. It should be noted that our method outperforms single-feature approaches on this dataset.

Table IV shows the accuracies obtained for each class when our approach is tested on the YouTube dataset. The table also shows comparison with other methods and highlights the number of features type used by each approach. We obtain an accuracy of $71.3 \%$ using our descriptor alone, and $72.5 \%$ when coupled with RANSAC for outlier rejection. Most of the confusion is observed between actions with similar motion, e.g. between biking, Horseback riding and walk dog, which involve translational motion; and between basketball shooting and volleyball spiking, which both involve the upward thrust of the hand, and often movement involving several players. Table IV compares our results to the approaches investigated by Liu et al [7]. It can be seen that, overall, our motion based approach out-performs Liu's motion and static features.

In order to assess the information gained by the descriptor using quaternary representation of node tests (equation 5) over binary tests, we also performed tests on the all the datasets using binary tests. Table V compares the results. It can be seen that quaternary tests consistently outperform 


\begin{tabular}{|l|c|c|c|c|c|}
\hline & \multicolumn{5}{|c|}{ Percent accuracy (\%) } \\
\hline Action & {$[7]$} & {$[26]$} & {$[27]$} & RMD & $\begin{array}{c}\text { RMD + } \\
\text { RANSAC }\end{array}$ \\
\hline \hline Biking & 73 & 58 & 75.2 & 75 & 75 \\
Diving & 81 & 79 & 95 & 87.2 & 87.9 \\
Golf & 86 & 66 & 95 & 93.4 & 91.3 \\
Soccer & 54 & 53 & 53 & 80.1 & 79.6 \\
Trampolining & 79 & 52 & 93 & 81.9 & 84.5 \\
Horse Riding & 72 & 81 & 73 & 75.8 & 77 \\
Basketball & 53 & 54 & 48.5 & 33 & 44.4 \\
Volleyball & 73.3 & 66 & 85 & 74.7 & 73.2 \\
Swing & 57 & 74 & 66 & 82.3 & 85.8 \\
Tennis & 80 & 59 & 77 & 57.1 & 57.6 \\
Walk Dog & 75 & 52 & 66.7 & 44 & 41.4 \\
\hline \#Features & 2 & 4 & 3 & 1 & 1 \\
\hline Average & 71.2 & 63.1 & 75.2 & 71.3 & 72.5 \\
\hline
\end{tabular}

TABLE IV

COMPARISON ON YOUTUBE ACTION DATASET.

\begin{tabular}{|c|c|c|}
\hline Dataset & Binary & Quaternary \\
\hline KTH & $87.9 \%$ & $91.2 \%$ \\
ADL & $75 \%$ & $84 \%$ \\
Kisses/Slaps & $67.7 \%$ & $74.6 \%$ \\
Youtube & $61.1 \%$ & $71.3 \%$ \\
\hline
\end{tabular}

TABLE V

COMPARISON OF RESULTS OBTAINED ON ALL FOUR DATASETS USING BINARY AGAINST QUATERNARY REPRESENTATION OF NODE TESTS.

binary tests, hence categorisation of actions benefits from quantizing the node test results with more symbols.

\section{CONCLUSION}

We presented a novel descriptor for actions in video which solely captures the dynamics of body movements via the relative distribution of motion-induced interest points. Our method assembles concatenated histograms of relative motion, which can be learnt by a discriminative classifier. When compared with approaches using multiple features, we show that, using one feature type, and quantizing the representation of the node tests with more symbols, performance consistent with state-of-the-art can be achieved. Our results are remarkable, considering that other methods used in our comparisons make use of three or four feature types. It should also be noted that no explicit appearance information is used. Hence, we show that body dynamics can be used as an effective descriptor of actions. We show consistent improvement in performance by discarding training examples that degrade the performance of the classifiers. These examples are discovered by our novel application of RANSAC in this domain.

For the KTH and Assisted Daily Living datasets, applying RANSAC results in few rejected outliers as the variations in action execution and camera setup are not significant compared to the YouTube and Kisses/Slaps datasets. For more complex datasets, a significant number of outliers per class will be expected. Hence, future work will investigate the application of RANSAC iteratively to training example to automatically discover different modes of action class examples that have been brought about by the variations. We expect that this will further improve action recognition in natural videos.

\section{ACKNOWLEDGEMENTS}

This work is supported by the EU FP7 Project Dicta-Sign (FP7/2007-2013) under grant agreement no 231135, and the EPSRC project Making Sense (EP/H023135/1).

\section{REFERENCES}

[1] R. Blake and M. Shiffrar, "Perception of human motion," Annual Review of Psychology, vol. 58, pp. 47-73, 2007.

[2] M. A. Fischler and R. C. Bolles, "Ransac: A paradigm for model fitting with applications to image analysis and automated cartography," Communications of the ACM, vol. 24, no. 6, 1981.

[3] C. Schuldt, I. Laptev, and B. Caputo, "Recognizing human actions: A local SVM approach," in ICPR, 2004

[4] J. Niebles, H. Wang, and L. Fei-Fei, "Unsupervised learning of human action categories using spatial-temporal words," IJCV, vol. 79, no. 3, pp. 299-318, 2008.

[5] P. Dollár, V. Rabaud, G. Cottrell, and S. Belongie, "Behavior recognition via sparse spatio-temporal features," in VS-PETS, 2005.

[6] I. Laptev, M. Marszalek, C. Schmid, and B. Rozenfeld, "Learning realistic human actions from movies," in CVPR, 2008.

[7] J. Liu, J. Luo, and M. Shah, "Recognizing realistic actions from videos "in the wild"," in CVPR, 2009.

[8] A. Gilbert, J. Illingworth, and R. Bowden, "Fast realistic multi-action recognition using mined dense spatio-temporal features," in ICCV, 2009.

[9] I. Laptev and T. Lindeberg, "Space-time interest points," in ICCV, 2003.

[10] G. Willems, T. Tuytelaars, and L. J. Van Gool, "An efficient dense and scale-invariant spatio-temporal interest point detector," in ECCV, 2008.

[11] P. Scovanner, S. Ali, and M. Shah, "A 3-dimensional sift descriptor and its application to action recognition," in Proc. Intl. Conf. on Multimedia, 2007, pp. 357-360.

[12] A. Kläser, M. Marszałek, and C. Schmid, "A spatio-temporal descriptor based on 3d-gradients," in BMVC, 2008.

[13] I. Laptev and P. Perez, "Retrieving actions in movies," in ICCV, 2007.

[14] M. Marszalek, I. Laptev, and C. Schmid, "Actions in context," in CVPR, 2009.

[15] M. Ryoo and J. Aggarwal, "Spatio-temporal relationship match: Video structure comparison for recognition of complex human activities," in ICCV, 2009.

[16] P. Matikainen, M. Herbert, and R. Sukthankar, "Representing pairwise spatial and temporal relations for action recognition," in ECCV, 2010.

[17] A. Kovashka and K. Grauman, "Learning a hierarchy of discriminative space-time neighborhood features for human action recognition," in CVPR, 2010.

[18] S. Savarese, A. DelPozo, J. Niebles, and L. Fei-Fei, "Spatial-temporal correlatons for unsupervised action classification," in WMVC, 2008.

[19] M. Ozuysal, P. Fua, and V. Lepetit, "Fast keypoint recognition in ten lines of code," in CVPR, 2007.

[20] R. Messing, C. Pal, and H. Kautz, "Activity recognition using the velocity histories of tracked keypoints," in ICCV, 2009.

[21] M. Rodriguez, J. Ahmed, and M. Shah, "Action mach: A spatiotemporal maximum average correlation height filter for action recognition," in CVPR, 2008.

[22] A. Gilbert, J. Illingworth, and R. Bowden, "Action recognition using mined hierarchical compound features," PAMI, vol. 99, no. PrePrints, 2010.

[23] A. Fathi and G. Mori, "Action recognition by learning mid-level motion features," in CVPR, 2008.

[24] L. Yaffet and L. Wolf, "Local trinary patterns for human action recognition," in ICCV, 2009.

[25] M. Raptis and S. Soatto, "Tracklet descriptors for action modeling and video analysis," in ECCV, 2010.

[26] M. Bregonzio, J. Li, S. Gong, and T. Xiang, "Discirminative topics modelling for action feature selection and recognition," in $B M V C$, 2010.

[27] N. Ikizler-Cinbis and S. Sclaroff, "Object, scene and actions: Combining multiple features for human action recognition," in ECCV, 2010. 